\title{
On the Riemann surface type of random planar maps
}

\author{
James T. Gill and Steffen Rohde
}

\begin{abstract}
We show that the (random) Riemann surfaces of the AngelSchramm uniform infinite planar triangulation and of Sheffield's infinite necklace construction are both parabolic. In other words, Brownian motion on these surfaces is recurrent. We obtain this result as a corollary to a more general theorem on subsequential distributional limits of random unbiased disc triangulations, following work of Benjamini and Schramm.
\end{abstract}

\section{Introduction and results}

Random maps (such as a random triangulation of the sphere, uniformly chosen among all triangulations with a fixed number of vertices) are of interest in probability, statistical physics, and combinatorics. Significant progress on their understanding has been gained in recent years based on new insights in combinatorics (specifically, bijections between maps and labelled trees [15], [6]), in probability (the papers [5], [1], [10] and many others) and in statistical physics (notably [7]).

The existence of certain local limits (e.g., the UIPT of Angel and Schramm [1]), and scaling limits (e.g., the work of J. F. Le Gall [10]) has been established, and first topological properties have been proved (such as one-endedness of the UIPT [1], and homeomorphicity of the scaling limits to the sphere [11], [13]). There are a large number of conjectures concerning further properties of these limits, and concerning their metric and geometric structure, e.g., [7], [3], and [17].

There are two well-developed mechanisms to give some sort of conformal structure to a discrete object such as a triangulation. One is by means of KoebeAndreev-Thurston circle packings: if the underlying graph $G$ has no loops and no double edges, then there is an essentially unique (up to Möbius transformation) collection of closed discs on the sphere, with pairwise disjoint interiors, such that the tangency graph (discs correspond to vertices of the graph, and tangencies of discs correspond to edges) is isomorphic to $G$. See [5] and [3] for this approach, and [19] or [14] for background on circle packings. A second and perhaps more

Mathematics Subject Classification (2010): Primary 30F20; Secondary 60B05.

Keywords: Riemann surface, random planar maps, uniformization. 
natural way works for all types of triangulations and is provided by a Riemann surface structure on the simplicial complex associated with the triangulation. Roughly speaking, one just glues equilateral triangles isometrically along their edges, see Section 2.2 below. In the limiting case of infinite triangulations, the complex is no longer compact, but in our setting it is still simply connected. By the uniformization theorem, the Riemann surface is thus either parabolic (conformally equivalent to the complex plane) or hyperbolic (equivalent to the disc). An equivalent characterization is that Brownian motion on the complex is either recurrent (in the parabolic case) or transient.

In this paper, we prove parabolicity of distributional limits of "unbiased" rooted triangulations (roughly, given an unrooted triangulation $T$, each face has the same probability of being the root; see Section 2.3 for details):

Theorem 1.1. Suppose that $(T, o)$ is a subsequential distributional limit of a sequence $\left(T_{n}, o_{n}\right)$ of random rooted finite unbiased disc triangulations (the limits taken with respect to the topology induced by the combinatorial distance $d_{c}$ of triangulations; see Section 2.1). Suppose further that $(T, o)$ has one end almost surely, and that the graph distance $d_{g r}\left(o_{n}, \partial T_{n}\right) \rightarrow \infty$ in law. Then the Riemann surface $R(T)$ is parabolic almost surely.

If $d_{g r}\left(o_{n}, \partial T_{n}\right)$ does not tend to $\infty$, the limiting Riemann surface has a boundary, and reflecting Brownian motion is still recurrent. An instructive example is the 7-regular graph, see Section 2.3 below.

There is no need to restrict to triangulations: Theorem 1.1 and its proof remain true with only minor changes for unbiased $m$-angulations, or slightly more generally by angulations with uniform bounds on the degrees of the faces.

Theorem 1.1 is similar to the result of Benjamini and Schramm [5] concerning the recurrence of a simple random walk on subsequential distributional limits of unbiased graphs. In fact, our method of proof is an adaptation of their method to the setting at hand. However, due to the use of circle packings, their proof only works under the additional assumption of uniformly bounded vertex degrees. Perhaps the most significant contribution of this paper is the realization that, when working with a Riemann surface, we do not need to make this extra assumption (we only use the trivial fact that the dual graph has bounded degree).

In [1], it was noted that the method of [5] can be adapted to show that the circle packing type of the UIPT is parabolic. It was also conjectured that simple random walk on the UIPT is recurrent a.s. As the vertex degree in the UIPT is a.s. unbounded, the method of [5] does not yield recurrence. While we are not able to prove this conjecture ${ }^{1}$, our theorem does show that Brownian motion on the UIPT is recurrent a.s. In other words, we prove:

Corollary 1.2. The Riemann surface associated with the UIPT is a.s. parabolic.

Notice that parabolicity of the UIPT is an implicit part (though much weaker) of Conjecture 7.1 in [7]. Another interesting random infinite surface, the "infinite

\footnotetext{
${ }^{1}$ After this article was submitted, this conjecture was proved in [8].
} 
necklace", was recently constructed by Scott Sheffield [18], see Figure 5 and Section 3.2 for our description. As another application of Theorem 1.1, we prove his conjecture concerning parabolicity of the associated Riemann surface:

Corollary 1.3. Brownian motion on the infinite necklace surface is almost surely recurrent.

We will now outline the proof of Theorem 1.1. How can one decide if a given triangulated Riemann surface $R$ is parabolic or hyperbolic? This type problem has been studied from many perspectives, but our approach is very simple and is motivated by the method of Benjamini and Schramm [5]. Fix a conformal map $\phi: R \rightarrow \mathbb{C}$. The direct analog of their method would be to associate with each vertex $v$ of the triangulation the half-flower $H_{v} \subset R$ (see Figure 1) and thus obtain a packing $P$ of the plane by topological discs $\phi\left(H_{v}\right)$ with tangency graph the triangulation. The role of the circle packing theorem is now played by the uniformization theorem, namely the existence of $\phi$. If it then were true that the discs $\phi\left(H_{v}\right)$ had bounded geometry (say, inradius and outradius comparable up to constants independent of $v$ ), then the parabolicity $\phi(R)=\mathbb{C}$ would be equivalent to showing that the set of the centers of the packing $\{\phi(v): v \in V\}$ has no finite accumulation point. Unfortunately, due to the unboundedness of the degree, we have not been able to show that the half-flowers have bounded geometry. But switching to the dual graph and considering the centers of the triangles instead of the vertices, we do obtain a test for parabolicity:

Proposition 1.4. Let $\phi$ be a conformal map of $R$ into $\overline{\mathbb{C}}$. Then $R$ is parabolic if and only if the following two conditions are satisfied: first, the set $\{\phi(c)\}$ of centers of triangles has precisely one accumulation point in $\overline{\mathbb{C}}$. And second, $d_{g r}(v, \partial T)=\infty$ for one (and hence all) vertices $v$.

The proof is given in Section 2.2 and consists in showing that the interstices of the half-flower packing have bounded geometry.

We are now in the situation of [5] and consider distributional limits $(G, o)$ of finite random unbiased graphs $\left(G_{n}, o_{n}\right)$. We reinterpret their Lemma 2.3 and proof of Proposition 2.2 in the following way, without the need to refer to packings. Assume that for each rooted graph we have an embedding $g_{n}$ of the set of vertices into the plane that is normalized in such a way that $g_{n}\left(o_{n}\right)=0$ and that the point of $\left\{g_{n}(v): v \in V_{n} \backslash\left\{o_{n}\right\}\right\}$ closest to 0 is at distance 1 from 0 . Assume further that any two embeddings $g_{n}$ and $g_{n}^{\prime}$ of the same (unrooted) graph differ only by a real dilation and complex translation. Finally, assume that $\left(G_{n}, o_{n}, g_{n}\right)$ converge to $(G, o, g)$ in the sense that $\left(G_{n}, o_{n}\right) \rightarrow(G, o)$ in distribution (with respect to the combinatorial distance defined in Section 2.1) and that furthermore $g_{n} \rightarrow g$ in distribution (with respect to a topology defined in Section 2.3).

Proposition 1.5. If $\left(G_{n}, o_{n}, g_{n}\right)$ converge to $(G, o, g)$ in the above sense, then the random set of points $g(G)$ has at most one accumulation point in $\mathbb{C}$ almost surely.

Notice that in Proposition 1.5, no assumption is made on the graphs $\left(G_{n}, o_{n}, g_{n}\right)$ apart from distributional convergence. Particularly, it is not assumed that the 
graphs are planar. However, for our application we have to work with the dual graph of the random triangulation, and embeddings of (centers of) faces rather than vertices. For the sake of clarity, in Proposition 2.5 of Section 2.3 we state and prove a version of Proposition 1.5 that is tailored to our applications. The proofs are identical and are basically in [5]. Theorem 1.1 is a consequence of Proposition 1.4 and (the variant of) Proposition 1.5. In order to apply Proposition 1.5, we need to prove existence of the limit $(G, o, g)$. An easy way to do this is by proving compact convergence of the conformal maps $\phi_{n}$ of the Riemann surfaces $R\left(T_{n}\right)$, which follows from Montel's theorem. To obtain Corollary 1.2, we approximate the UIPT by an unbiased sequence of disc triangulations, simply by removing a randomly chosen triangle. See Section 3.1 for details. Finally, to prove Corollary 1.3 , we show that the boundary of the random disc with $n$ triangles has size about $\sqrt{n}$ (Lemma 3.5), so that the distance of the root to the boundary stays bounded with probability about $1 / \sqrt{n}$ (Lemma 3.6).

Acknowledgements. The authors would like to thank Itai Benjamini and Nicolas Curien for helpful comments on a draft of this paper and the anonymous referee for a careful reading of the paper and some helpful suggestions. When this research was completed the first author of this note was also in the Department of Mathematics at the University of Washington.

\section{Packings, triangulations, and Riemann surfaces}

In this section, we will describe the Riemann surface associated with a triangulation via gluing of equilaterals, and provide a simple criterion for parabolicity of such a surface.

\subsection{Triangulations}

We adopt the terminology and definitions of the important papers [5] and [1] on random planar triangulations. Here we restrict ourselves to very briefly listing and explaining the key terms, and refer the reader to the well written and nicely illustrated Section 1.2 in [1] for motivation and details.

An embedded triangulation $T$ consists of a finite or infinite connected graph $G$ embedded in the sphere $\overline{\mathbb{C}}$, together with a subset of the triangular faces (that is, connected components of $\mathbb{C} \backslash G$ whose boundary meets precisely three edges of $G$ ). We will drop the term embedded, and we will always assume that the triangulation is locally finite, that is, every point in the support $S(T)$ (the union of $G$ and all the triangles in $T$ ) has a neighborhood in $\overline{\mathbb{C}}$ that intersects only a finite number of elements of $T$. We use $F(T)$ to denote the collection of open faces in the triangulation $T$. We will only be concerned with the number of elements in $F(T)$. Two triangulations $T$ and $T^{\prime}$ will be considered equivalent if there is an orientation preserving homeomorphism of their supports corresponding $T$ and $T^{\prime}$. See Definitions 1.1 and 1.5 in [1]. There are different types of triangulations, according to what types of graphs we allow. We will only consider type II triangulations where $G$ has no loops but possibly multiple edges, and type III triangulations 
where $G$ has neither loops nor multiple edges (Definitions 1.2 and 1.3 in [1]). A rooted triangulation $(T, o)$ is a triangulation $T$ together with an oriented triangular face $o=(x, y, z)$ of $T$, called the root. The vertex $x$ is the root vertex and $(x, y)$ is the root edge.

We call $T$ a disc triangulation if the support is simply connected. For finite triangulations, this implies that the complement $\overline{\mathbb{C}} \backslash S(T)$ is an $m$-gon for some $m$, so that $T$ is a triangulation of an $m$-gon. A vertex $v$ is an interior resp. boundary vertex if $v$ belongs to the interior (resp. boundary) of $S(T)$. Denote $\partial T$ the set of boundary vertices (which is empty if $S(T)$ is open). An infinite triangulation is a disc triangulation if it has no boundary vertices and is one ended, i.e., the deletion of any finite subgraph leaves only one infinite connected component.

Denote by $d_{g r}\left(v_{1}, v_{2}\right)$ the graph distance on $T$ and write $d_{g r}(v, \partial T)$ for the minimal graph distance between $v$ and any point $v^{\prime} \in \partial S(T)$ (if there is no such $v^{\prime}$, we set $\left.d_{g r}(v, \partial T)=\infty\right)$. Define the combinatorial distance between two rooted triangulations $(T, o)$ and $\left(T^{\prime}, o^{\prime}\right)$ as

$$
d_{c}\left((T, o),\left(T^{\prime}, o^{\prime}\right)\right)=1 /(k+1)
$$

where $k$ is the largest radius $r$ for which the combinatorial balls $B_{r}$ and $B_{r}^{\prime}$ around the root vertices $v, v^{\prime}$ are equivalent, where equivalency now also requires that the root is preserved. (The inductive definition of $B_{r}$, given in [1], Definition 4.3, is that $B_{0}$ is the root vertex and $B_{r+1}$ consists of all triangles incident to a vertex of $B_{r}$, together with all vertices and edges of these triangles).

We will mostly deal with disc triangulations and their limits. In general, it is not true that limits of disc triangulations are disc triangulations.

Lemma 2.1. If $\left(T_{n}, o_{n}\right)$ is a sequence of disc triangulations converging to a triangulation $(T, o)$ in $d_{c}$, if $d_{g r}\left(o_{n}, \partial T_{n}\right) \rightarrow \infty$, and if $T$ has one end, then $T$ is a disc triangulation.

We leave the proof of the lemma as an exercise to the reader. None of the assumptions of the lemma can be omitted. To see this, consider appropriate sequences of triangulations of the cylinders $S^{1} \times[0, n]$ and $S^{1} \times[-n, n]$, for instance by restricting the planar triangular lattice to a strip. Both are disc triangulations converging to triangulations of punctured discs (namely a half-infinite and a biinfinite cylinder). The first limit has one end but $d_{g r}\left(o_{n}, \partial T_{n}\right) \nrightarrow \rightarrow \infty$, the second limit has two ends (though $d_{g r}\left(o_{n}, \partial T_{n}\right) \rightarrow \infty$ ).

\subsection{Riemann surfaces}

With each triangulation we associate a metric space $\left(M(T), d_{M}\right)$, by regarding each triangle of $T$ as an equilateral triangle and defining the distance between two points as the length of the shortest path joining them. In other words, $M(T)$ is obtained by gluing equilateral triangles according to their adjacency pattern in $T$.

This metric space can be equipped with a compatible Riemann surface structure by defining coordinate charts as follows. If $x$ is an interior point of a triangle, simply use that triangle as the chart, view it as a subset of the plane, and use 
the identity map as the projection. Similarly, if $x$ is interior to an edge, simply place the two adjacent triangles in the plane and again use the identity as the projection. (If $x$ is in the boundary of the support, there will be only one triangle and we obtain a bordered Riemann surface). Finally, if $x$ is a vertex of degree $n$, unless $n=6$ we cannot place the triangles in the plane so that they form a neighborhood of $x$. Nevertheless, we take for the chart the union of $x$ with the edges and faces adjacent to $x$. Loosely speaking, we set $x=0$ and take the map $z \mapsto z^{6 / n}$ as the coordinate map. More precisely, if $\Delta_{1}, \Delta_{2}, \ldots, \Delta_{n}$ denote the equilateral triangles which meet at $x$ in cyclic order, we may place them in the plane so that $\Delta_{j}$ is the triangle with vertices $0, e^{2 \pi i(j-1) / 6}, e^{2 \pi i j / 6}$. Then we define the coordinate map for $z=r e^{i t} \in \Delta_{j}$ by $\phi(z)=r^{6 / n} e^{6 t i / n}$, using the convention that $2 \pi(j-1) / 6<t<2 \pi j / 6$ on $\Delta_{j}$. Finally, we set $\phi(0)=0$ and notice that $\phi$ is a homeomorphism between a neighborhood of $x$ in $M$ and a neighborhood of 0 in $\mathbb{C}$. It is obvious that coordinate changes are analytic, so that this indeed defines a Riemann surface.

Following [5] (but using triangles instead of vertices for roots, as in [1]), we call a random rooted triangulation $(T, o)$ unbiased if the law $\mu$ of $(T, o)$ is in the closed convex hull of the measures $\mu_{H}$ which are defined for finite triangulations $H$ as the uniform measure on rooted triangulations $(H, o)$ where $o$ ranges over all possible triangles of $H$. Informally, $(T, o)$ is unbiased if given $H$ the root $o$ is uniformly distributed among the oriented triangles.

We now state our parabolicity criterion for Riemann surfaces of random triangulations. It is the Riemann surface analog of Theorem 1.1 of [5], the recurrence of simple random walk on distributional limits of unbiased finite planar graphs with uniformly bounded degrees. Notice that we allow unbounded degrees, and that we assume existence of a subsequential limit.

Theorem 1.1. Suppose that $(T, o)$ is a subsequential distributional limit of a sequence $\left(T_{n}, o_{n}\right)$ of random rooted finite unbiased disc triangulations (the limit taken with respect to the topology induced by the combinatorial distance $d_{c}$ of triangulations). Suppose further that $(T, o)$ has one end almost surely, and that $d_{g r}\left(o_{n}, \partial T_{n}\right) \rightarrow \infty$ in law. Then the Riemann surface $R(T)$ is parabolic a.s.

Put differently, under the assumptions of the theorem, Brownian motion on any distributional limit Riemann surface is recurrent a.s. If we omit the assumption $d_{g r}\left(o_{n}, \partial T_{n}\right) \rightarrow \infty$, the limiting Riemann surface could have a boundary. In this case, reflecting Brownian motion would still be recurrent. The crucial condition on unbiasedness can be somewhat weakened. For instance, it would be enough to assume that, given a finite triangulation $T$, for "most" possible roots $o$ the probability that $o_{n}=o$ is comparable to the reciprocal of the number of faces of $T$. Indeed, our proof goes through without modifications if unbiasedness is replaced by the existence of a constant $C$ such that for each $\varepsilon>0$ there is $n_{0}$ such that for $n \geq n_{0}$, if $U_{n}(T)$ denotes the set of those directed triangles $o$ of $T$ for which

$$
\frac{1}{C|F(T)|} \leq \mathbb{P}\left[o_{n}=o \mid T_{n}=T\right] \leq \frac{C}{|F(T)|},
$$


then

$$
\mathbb{P}\left[o_{n} \in U(T) \mid T_{n}=T\right] \geq 1-\varepsilon .
$$

The recurrence proof [5] used circle packings and was based on the following result from [9]: if a collection of discs $D_{v} \subset \mathbb{C}$ with pairwise disjoint interiors has no accumulation point in the plane, and if its tangency graph $G$ has uniformly bounded vertex degree, then $G$ is recurrent. We will proceed similarly, by associating with each triangulation a packing of topological discs in the plane. One of the differences, necessitated by our need to work with unbounded degrees, is that we work with a packing of the dual graph rather then the original graph (in circle packings, this would correspond to the collection of interstices rather than the collection of discs). First we give some definitions. Let $v$ be a vertex of $T$ of degree $\operatorname{deg} v=n$. The flower $F_{v}=F_{v}(T) \subset R$ of $v$ is the union of the $n$ equilateral triangles $\left(v, v_{i}, v_{i+1}\right)$ which meet at $v$, together with the edges. Similarly, the half-flower $H_{v}$ is the $n$-gon formed by the $\operatorname{deg}(v)$ equilateral triangles $\left(v, v_{i}^{\prime}, v_{i+1}^{\prime}\right)$, where $v_{i}^{\prime}$ is the midpoint between $v$ and $v_{i}$, see Figure 1 . The interstice $I_{f}$ of a triangular face $f$ with vertices $\left(v_{1}, v_{2}, v_{3}\right)$ is the equilateral subtriangle whose vertices are the midpoints of the edges, see Figure 2. We denote by $c_{f}$ the center of a face. The interstices and the half-flowers form a decomposition of the surface. The names are borrowed from the theory of circle packings (see [19] or [14]).
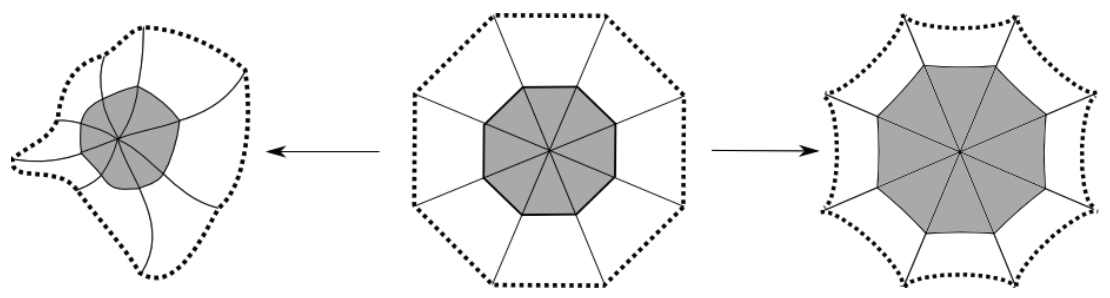

Figure 1. The flower, half-flower (shaded), and their images under a uniformizing map (left) and under the coordinate map (right).

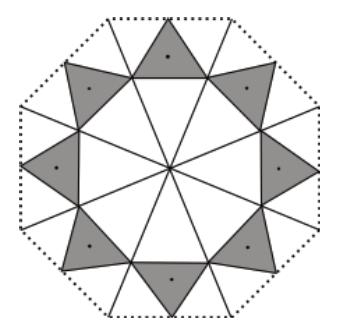

Figure 2. The same flower as in Figure 1 but with the interstitial triangles shaded.

For the remainder of this section, we assume that $T$ is a disc triangulation so that $R$, the Riemann surface associated with $T$, is simply connected. By the Koebe uniformization theorem, there is a conformal homeomorphism into the sphere $\overline{\mathbb{C}}$. The following simple criterion for parabolicity is key to our approach. 
Proposition 1.4. Let $\phi$ be a conformal map of $R$ into $\overline{\mathbb{C}}$. Then $R$ is parabolic if and only if the following two conditions are satisfied: first, the sequence of centers of interstices $\phi\left(c_{f}\right)_{f \in F(T)}$ has precisely one accumulation point in $\overline{\mathbb{C}}$. And second, $d_{g r}(v, \partial T)=\infty$ for one (and hence all) vertices $v$.

Remark 2.2. The proposition also holds if we replace the condition of "precisely one accumulation point in $\overline{\mathbb{C}}$ " with "countably many accumulation points in $\overline{\mathbb{C}}$ ". A variant with finitely many accumulation points is what we will eventually use in the proof of Theorem 1.1.

The condition on the centers of interstices can be replaced by the condition that the sequence of interstices $\phi\left(I_{f}\right)_{f \in F(T)}$ has at most one accumulation point in $\overline{\mathbb{C}}$. We believe that the condition is also equivalent to requiring that the half-flowers, or their centers, have only one accumulation point. This would be the most direct analog of the notion of "circle packing parabolicity" of He and Schramm, [9], and the aforementioned approach in [5]. Since we do not assume the degrees of the vertices to be bounded, we have no control over the geometry of the half-flowers. Indeed, it is easy to see that the hyperbolic diameter of the half-flowers within the combinatorial ball $B_{1}$ tends to infinity as the degree of the root vertex tends to infinity. To see what could happen when no bounded geometry assumption is made, consider Figure 3.

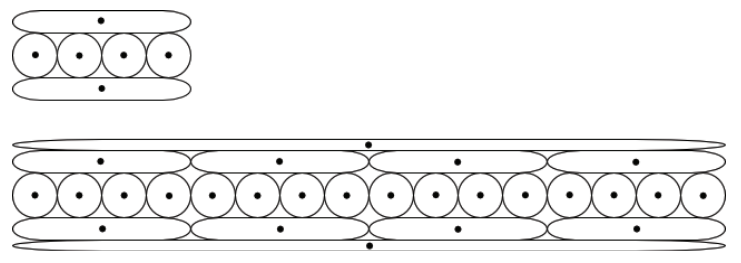

FiguRE 3. A packing of a hyperbolic graph by sets of unbounded geometry

It shows the first two steps of an iterative construction of a (deterministic) packing of a hyperbolic (transient) graph (it is similar in spirit to the image of Figure 4 under the homeomorphism $z \mapsto z /(1-|z|)$ mapping the unit disc to the plane). The packing has two infinite lines of accumulation, but it is easy to associate with each of the packed sets a point "well within" the set such that this collection of points has no accumulation point in the plane. The problem is that the points no longer properly "represent" the topological discs because there is no uniform assumption on the geometry of the discs. We get around this problem by considering the interstices. Notice that in Figure 3 the interstices do not have bounded geometry. Our proof of the proposition relies crucially on the fact that the Riemann surface interstices have bounded geometry, independently of the degrees of the vertices.

Lemma 2.3. There is a constant $C$ such that for all simply connected open $R$ as above, all faces $f$ of $R$ with $d_{g r}(f, \partial R) \geq 2$, and all conformal maps $\phi: R \rightarrow \mathbb{C}$,

$$
B\left(\phi\left(c_{f}\right), \frac{1}{C} \operatorname{diam} \phi\left(I_{f}\right)\right) \subset \phi\left(I_{f}\right) \subset B\left(\phi\left(c_{f}\right), C \operatorname{diam} \phi\left(I_{f}\right)\right) .
$$


If $f^{\prime}$ is a face adjacent to $f$, then

$$
\frac{1}{C} \leq \frac{\left|\phi\left(c_{f}\right)-\phi\left(c_{f^{\prime}}\right)\right|}{\operatorname{diam} \phi\left(I_{f}\right)} \leq C .
$$

If furthermore $R$ is hyperbolic, then the hyperbolic diameter of $I_{f}$ satisfies

$$
\operatorname{diam}_{\text {hyp }}\left(I_{f}\right)<C \text {. }
$$

Proof. Consider the union $U$ of $f$ with the triangles sharing an edge with $f$. Then $U$ consists of either four triangles, or (in the presence of double edges) $U$ consists of three triangles. In both cases, the interstice $I_{f}$ and the line segment $\left[c_{f}, c_{f^{\prime}}\right]$ are compact subsets of $U$ and thus of finite hyperbolic diameter within $U$. If $\psi$ is a conformal map of $U$ onto the unit disc, such that $c_{f}$ maps to 0 , then (2.1) and (2.2) are trivially satisfied since there are only two such maps $\psi$. Now standard applications of the Koebe distortion theorem give (2.1) and (2.2). Since the hyperbolic distance decreases as the domain increases (essentially Schwarz's Lemma), $I_{f}$ and $\left[c_{f}, c_{f^{\prime}}\right]$ have uniformly bounded hyperbolic diameter independent of the triangulation. This proves (2.3).

Proof of Proposition 1.4. Since $R$ is not conformally $\overline{\mathbb{C}}$, we may assume that $\phi(R) \subset \mathbb{C}$.

If $R$ is parabolic, then $d_{g r}(v, \partial T)=\infty$, for otherwise there would be a boundary edge at finite combinatorial distance from $v$ and Brownian motion would be transient. Also, in this case $\phi$ is a proper homeomorphism between $R$ and $\mathbb{C}$, so that $\infty$ is the only limit point.

To show the converse, assuming that $R$ is hyperbolic and that $d_{g r}(v, \partial T)=\infty$ we will show that the sequence $\phi\left(c_{f}\right)$ has countably many accumulation points. Pick a point $w \in \partial \phi(R)$ (the boundary with respect to the Euclidean metric) such that there is a curve $\gamma$ in $\phi(R)$ joining $\phi(v)$ and $w$. Since $\phi(R)$ is hyperbolic, $\partial \phi(R)$ is nonempty and connected, the set of such $w$ is of full harmonic measure, hence uncountable (for instance, the points in $\partial \phi(R)$ closest to some interior point will do). The curve $\phi^{-1}(\gamma)$ eventually leaves every compact set and therefore has to meet infinitely many interstices $I_{1}, I_{2}, \ldots$ In particular, there is a sequence of points $z_{j} \in I_{j}$ such that $\phi\left(z_{j}\right) \rightarrow w$. By (2.3) and the Koebe distortion theorem, it follows that $\operatorname{diam} \phi\left(I_{f}\right) \leq C^{\prime}\left|w-\phi\left(z_{j}\right)\right|$, so that $\phi\left(c_{j}\right) \rightarrow w$ as well.

\subsection{Center embeddings}

In this section, we develop a variant of the Benjamini-Schramm technique, [5], suitable for our needs. See [4] for a different (but related) variant. One of the key ideas in [5] was to show that the centers of the circle packings associated with a distributional limit of unbiased random triangulations of the sphere have only one limit point almost surely. In the case of uniformly bounded vertex degrees, this implied recurrence of the simple random walk, by [9] and [12].

With our parabolicity criterion Proposition 1.4 in mind (and thinking of the Riemann surface interstice-packing as a substitute for the circle packings), we are 
interested in finite or countably infinite sets of points $\phi\left(c_{f}\right)_{f \in F(T)}$, together with the combinatorics of the triangulation. We thus make the following:

Definition 2.4. A center embedding is a triple $E=(T, o, g)$, where $(T, o)$ is a rooted triangulation and $g: F(T) \rightarrow \mathbb{C}$ an injective map. We also require $g$ to be normalized by $g(o)=0$ and such that the closest point is of distance 1 ,

$$
\inf _{f \neq o}|g(f)|=1 \text {. }
$$

We equip the space $\mathcal{E}$ of normalized center embeddings with a topology in such a way that

$$
\left(T_{n}, o_{n}, g_{n}\right) \rightarrow(T, o, g)
$$

if and only if both $\left(T_{n}, o_{n}\right) \rightarrow(T, o)$ in the graph metric, and $g_{n}(f) \rightarrow g(f)$ in the Hausdorff metric for each face $f \in T$. Using Polish notation for the Hausdorff distance, $\left|g_{n}(f)-g(f)\right|$, and writing $d=d_{g r}\left((T, o),\left(T^{\prime}, o^{\prime}\right)\right)$, this topology is easily seen to be generated by the metric

$$
d_{\mathcal{E}}\left((T, o, g),\left(T^{\prime}, o^{\prime}, g^{\prime}\right)\right)=d+\sum_{n=0}^{d^{-1}-1} \frac{1}{2^{n+1}\left|B_{n}\right|} \sum_{f \in B_{n}} \frac{\left|g(f)-g\left(f^{\prime}\right)\right|}{1+\left|g(f)-g\left(f^{\prime}\right)\right|},
$$

where $B_{n}$ denotes the ball of radius $n$ in $T,\left|B_{n}\right|$ denotes the number of faces of $B_{n}$, and where $f^{\prime}$ denotes the face in $T^{\prime}$ corresponding to $f \in T$. It is not hard to check that $\left(\mathcal{E}, d_{\mathcal{E}}\right)$ is separable metric space. Slightly abusing standard terminology, we call two functions $g$ and $g^{\prime}$ similar if $g^{\prime}=a g+b$ with real $a>0$ and complex $b$. Thus each function $g: F(T) \rightarrow \mathbb{C}$ is similar to a normalized one.

Proposition 2.5. Suppose that $E_{n}$ is a sequence of random unbiased finite center embeddings such that the (unrooted) triangulation $T_{n}$ determines the embedding (in the sense that if $T$ and $T^{\prime}$ are equivalent as planar graphs, then the embeddings $g$ and $g^{\prime}$ are similar). Then for every distributional subsequential limit $E$ of $E_{n}$, the (random) set $g(F(T))$ has at most one limit point almost surely.

By a random center embedding we mean a probability measure $\mathbb{P}$ on $\mathcal{E}$, and unbiasedness refers to the induced measure on rooted graphs (given a finite graph, each face has the same probability of being the root). Notice that the existence of a limit is an assumption, not a conclusion.

Example 2.6. Instructive examples are the 6-regular triangular lattice and the 7-regular lattice and the corresponding centers of Riemann surface interstices $E_{\infty}$ (or circle packings $\mathbf{P}_{\infty}$ ). If $E_{n}$ is obtained from the combinatorial ball $B_{n}$ by uniformly choosing a root face, then the proposition applies in both the 6 - and 7-regular cases, and the limit $E$ (which is easily seen to exist in both cases) has no finite limit point. In Figure 4 below, we show two versions of circle packings of the 7-regular lattice. The first is an approximation to $E_{\infty}$. The set $E_{\infty}\left(\operatorname{resp} . \mathbf{P}_{\infty}\right)$ clearly has infinitely many limit points in the 7-regular case. This appears to be a contradiction to our proposition. It is not. The explanation is that $E_{\infty} \neq E$ 
in the 7-regular case. The drawing on the right of Figure 4 is a better picture for the limit embedding $E$. It is simply what the figure on the left becomes when one normalizes with respect to a boundary circle. Moreover, in this example, Theorem 1.1 does not apply: because of the linear isoperimetric inequality, the distance of the root to the boundary does not tend to infinity in law.
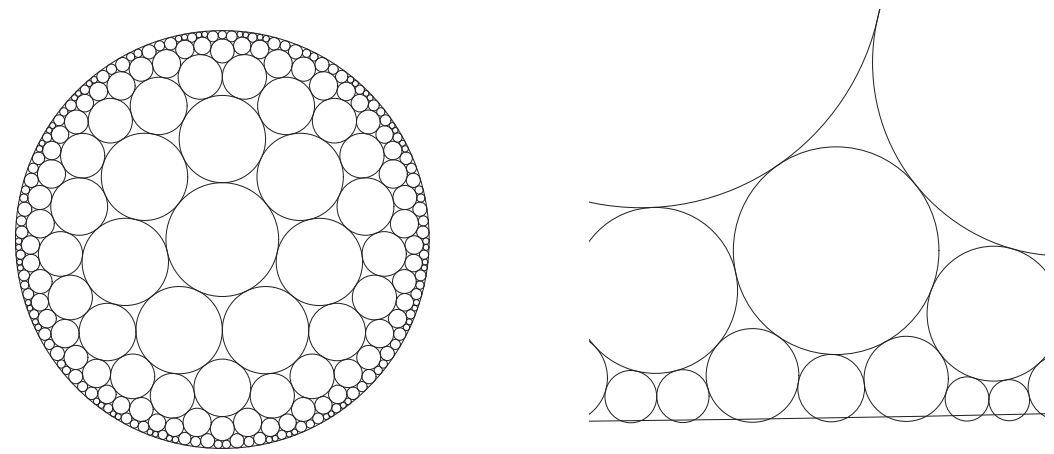

FiguRE 4. Approximations of 7-regular lattice circle packing, two points of view.

As mentioned in the introduction, the proof of Proposition 2.5 is essentially the same as the proof of Proposition 2.2 in [5]. Since our setup is different, we go through the details for the convenience of the reader. The key technical result is the following magical Lemma 2.3 from [5] (also see [4] for a nice exposition and generalizations to higher dimensions). It can be viewed as a quantitative statement to the effect that every finite planar set "looks like" it has at most one accumulation point, when viewed from "most" of the points. Here is the precise statement. Let $V \subset \mathbb{C}$ be a finite set of points (in our application, $V$ will consist of the marked points in the packings). For $v \in V$, the isolation radius is defined as

$$
\rho_{v}:=\inf \{|v-w|: w \in V \backslash\{v\}\} .
$$

Given $\delta>0, s>0$, and $v \in V$, say that $v$ is $(\delta, s)$-supported if

$$
\inf _{p \in \mathbb{C}}\left|V \cap\left(D\left(v, \rho_{v} / \delta\right) \backslash D\left(p, \rho_{v} \delta\right)\right)\right| \geq s .
$$

Lemma A. (Lemma 2.3 in [5]) For every $\delta \in(0,1)$ there is a constant $c=c(\delta)$ such that for every finite set $V \subset \mathbb{C}$ and every $s \geq 2$ the proportion of $(\delta, s)$-supported points in $V$ is less than $c / s$.

Proof of Proposition 2.5. The proof is by contradiction. Suppose that a distributional limit $E=(T, o, g)$ has two or more accumulation points with positive probability. Then there is $\delta>0$ and two points $p_{1}, p_{2} \in D(0,1 / \delta),\left|p_{1}-p_{2}\right| \geq 3 \delta$, such that the set of embeddings with accumulation points in $D\left(p_{1}, \delta\right)$ and in $D\left(p_{2}, \delta\right)$ 
has positive probability $\alpha>0$. For $k>0$, denote by $\mathcal{O}_{k}$ the set of all embeddings such that $B\left(p_{1}, \delta\right)$ and $B\left(p_{2}, \delta\right)$ contain at least $s$ points from the embedding corresponding to faces in $B_{k}$. Then there is $k=k_{s}$ such that the event $T \in \mathcal{O}_{k_{s}}$ has probability at least $\alpha / 2$. Since $\mathcal{O}_{k_{s}}$ is open with respect to the metric $d_{\mathcal{E}}$, the distributional convergence implies that the liminf of the probability of $T_{n} \in \mathcal{O}_{k_{s}}$ is greater than or equal to $\alpha / 2$. But the magical Lemma A, with $\delta$ fixed, implies 0 (i.e., the image of the center of $\left.T_{n}\right)$ is $(\delta, s)$-supported. Hence as $T_{n}$ is unbiased one deduces that $T_{n} \in \mathcal{O}_{k_{s}}$ has probability at most $c(\delta) / s$, a contradiction when $s>\alpha /(2 c(\delta))$.

Remark 2.7. For the purpose of proving parabolicity of the UIPT and the necklace, we could work in the perhaps more intuitive setting of packings of topological discs, instead of center packings. That is, we could consider finite or infinite collections $\mathbf{P}=\left\{P_{v}: v \in V\right\}$ of closed topological discs $P_{v} \subset \mathbb{C}$ with pairwise disjoint interiors, together with a collection of interior points $x_{v} \in P_{v}$.

Then the appropriate topology is induced by the metric

$d_{\mathcal{P}}\left(\mathbf{P}, \mathbf{P}^{\prime}\right)=d_{c}\left(P, P^{\prime}\right)+\sum_{n=0}^{d_{c}\left(P, P^{\prime}\right)^{-1}} \frac{1}{2^{n+1}\left|B_{n}\right|} \sum_{v \in B_{n}}\left(\frac{\left|x_{v}-x_{v^{\prime}}\right|}{1+\left|x_{v}-x_{v^{\prime}}\right|}+\frac{d_{H}\left(P_{v}, P_{v^{\prime}}\right)}{1+d_{H}\left(P_{v}, P_{v^{\prime}}\right)}\right)$

where $d_{H}$ is Hausdorff distance. The laws of the interstice-packings converge even in this finer topology. However, Proposition 2.5 is more general and the assumptions are easier to verify.

\subsection{Proof of Theorem 1.1}

With an eye toward Proposition 2.5, we first associate a center embedding with a rooted triangulation. For each finite (unrooted) disc triangulation $T$, fix a single conformal map

$$
\phi_{T}: R(T) \rightarrow \mathbb{C}
$$

Given a rooted finite disc triangulation $\left(T_{n}, o_{n}\right)$, define a normalized conformal map by

$$
\phi_{n}=a \phi_{T_{n}}+b
$$

where $a>0$ and $b \in \mathbb{C}$ are chosen so that

$$
\phi_{n}\left(c_{o_{n}}\right)=0
$$

and

$$
\inf _{f} \operatorname{dist}\left(0, \phi_{n}\left(I_{f}\right)\right)=1 \text {, }
$$

where the infimum is over all faces $f \in F(T) \backslash\{o\}$. Notice that

$$
\inf _{f} \operatorname{dist}\left(0, \phi_{n}\left(I_{f}\right)\right)=\min _{f \in B_{2}} \operatorname{dist}\left(0, \phi_{n}\left(I_{f}\right)\right)
$$


if $d_{g r}(o, \partial T) \geq 2$. Now assume that the unbiased disc triangulations $\left(T_{n}, o_{n}\right)$ converge in distribution to $(T, o)$. We claim that the sequence $\left(T_{n}, o_{n}, \phi_{n}\right)$ has a subsequential distributional limit $(T, o, \phi)$ with respect to compact convergence, and that $\phi$ is a normalized conformal map of $(T, o)$. To see this, we first prove tightness of the law $\mathbb{P}_{n}$ of $\left(T_{n}, o_{n}, \phi_{n}\right)$. As $\left(T_{n}, o_{n}\right) \rightarrow(T, o)$ in distribution, if $\epsilon>0$ is given, we can choose $L_{r}$ such that the set $A$ of all triples $(T, o, \phi)$, with

$$
\max _{v \in B_{r}(o)} \operatorname{deg} v<L_{r}
$$

for all $r$ and $\phi$ a normalized map of $(T, o)$, has

$$
\mathbb{P}_{n}[A] \geq 1-\varepsilon
$$

for each $n$. To see that $A$ is compact, fix a (deterministic) sequence $\left(T_{n}, o_{n}, \phi_{n}\right) \in A$ and assume without loss of generality that $\left(T_{n}, o_{n}\right)$ already converges (as there are a bounded number of possible $r$-neighborhoods of the root for each $r \geq 1$, every sequence of triangulations in $A$ has a subsequence that converges with respect to $\left.d_{g r}\right)$. Then fix $r$ and consider the sequence $\phi_{n}$ restricted to $B_{r}$. For $n$ large enough $B_{r}\left(T_{n}\right)$ is isomorphic to $B_{r}(T)$, and we can assume that all $\phi_{n}$ are defined on the same subset $B_{r}$ of $T$. Denote by $c=c_{o}$ the center of the root triangle, and by $c^{\prime}$ the center of a fixed neighboring triangle of the root. Since the interstices have disjoint interiors, it follows from (2.1) and (2.6) that

$$
\operatorname{diam} \phi_{n}\left(I_{o}\right) \leq C \inf _{f \neq o} \operatorname{dist}\left(0, \phi_{n}\left(I_{f}\right)\right)=C .
$$

On the other hand, (2.2) implies

$$
1 \leq\left|\phi_{n}(c)-\phi_{n}\left(c^{\prime}\right)\right| \leq C \operatorname{diam} \phi_{n}\left(I_{o}\right),
$$

and we obtain

$$
1 \leq\left|\phi_{n}\left(c^{\prime}\right)\right| \leq C^{2}
$$

Since the family $\phi_{n}$ omits the values $0, \phi_{n}\left(c^{\prime}\right)$ and $\infty$ on $B_{r} \backslash\left\{o, o^{\prime}\right\}$, Montel's theorem implies that it is a normal family. By a diagonal process we can extract a subsequence that converges compactly on every $B_{r}$, thus on $R(T)$. It follows from (2.9) that the limit is nonconstant, and by (2.7) it is a normalized conformal map of $R(T)$. Thus $A$ is compact, and $\mathbb{P}_{n}$ is tight. Now Prokhorov's theorem shows the existence of subsequential distributional limits, where $\phi$ is a normalized conformal map of $(T, o)$. Along this subsequence, $E_{n}=\left(T_{n}, o_{n}, g_{n}\right)$ satisfies the assumptions of Proposition 2.5, where $g_{n}$ denotes the restriction of $\phi_{n}$ to the centers $c_{f}$ of the faces $f$ of $T_{n}$, renormalized to satisfy (2.4). Indeed, if $T_{n}$ and $T_{n}^{\prime}$ are equivalent as unrooted triangulations, then $\phi_{n}=a \phi+b$ and $\phi_{n}^{\prime}=a^{\prime} \phi+b^{\prime}$ by $(2.5)$ so that $g_{n}$ and $g_{n}^{\prime}$ are similar. It follows from Proposition 2.5, that the sequence of centers has only one accumulation point in $\mathbb{C}$. Hence it has at most two accumulation points in $\overline{\mathbb{C}}$. Since $d_{g r}(o, \partial T)=\infty$, Proposition 1.4 (more precisely Remark 2.2) implies that $R(T)$ is parabolic a.s. 


\section{Applications}

\subsection{Parabolicity of the UIPT}

Following Angel and Schramm, denote by $\mathcal{T}_{n} \subset \mathcal{T}$ the set of all rooted triangulations of $S^{2}$ with $n$ vertices, and denote by $\tau_{n}$ the uniform measure on $\mathcal{T}_{n}$. More precisely, for $j=2$ and $j=3$ denote by $\tau_{n}^{j}$ the uniform measure on the the set of triangulations of type II (no loops, but double edges are allowed) and of type III (neither loops nor double edges), and let $\tau_{n}$ denote either of the two measures. The following statements are proved in [1]:

Theorem B. (Theorem 1.8, Theorem 1.10, and Corollary 4.5 in [1]) The measures $\tau_{n}$ converge in distribution to a measure $\tau$ supported on infinite triangulations in $\mathcal{T}$, called the uniform infinite planar triangulation (UIPT). Samples from $\tau$ have almost surely one topological end. Moreover, for each fixed $r$, the law of the maximal vertex degree in the ball of radius $r$ (centered at the root) is tight.

Proof of Corollary 1.2. By the Euler formula, a triangulation of the sphere with $n$ vertices has $2 n-4$ faces and $3 n-6$ edges. In order to apply Theorem 1.1 , we need to approximate the UIPT by finite unbiased disc triangulations with $d\left(o_{n}, \partial T_{n}\right) \rightarrow \infty$. To this end, let $S_{n}$ be an unrooted triangulation chosen according to the distribution induced by $\tau_{n}$ (which is not quite uniform due to triangulations with nontrivial symmetries). Choose two of the $2 n-4$ faces $\Delta$ and $o$ uniformly at random. Then $\left(S_{n}, o\right)$ is a uniform rooted triangulation, and $o$ is uniformly distributed over the triangles of $S_{n} \backslash \Delta$. Now let $\left(T_{n}, o_{n}\right)$ be the random rooted disc triangulation obtained by removing $\Delta$ from $S_{n}$ and by setting $o_{n}=o$. By tightness of the number of vertices in the ball $B_{r}$, one finds that

$$
d_{g r}\left(o_{n}, \partial T_{n}\right)=d_{g r}\left(o, \partial\left(S_{n} \backslash \Delta\right)\right) \rightarrow \infty
$$

in distribution. It follows that $\left(T_{n}, o_{n}\right)$ and $\left(S_{n}, o_{n}\right)$ have the same distributional limit, namely the UIPT. By Theorem B, the limit is one-ended almost surely, so that all the assumptions of Theorem 1.1 are satisfied and the UIPT is parabolic.

\subsection{The infinite necklace}

3.2.1. Setup. We begin with a description of the "necklace" construction of random maps due to Scott Sheffield, and then show that the assumptions of Theorem 1.1 are satisfied.

At each time $j$ of the inductive construction, we will have a triangulation of a disc $D_{j}$ in the closed upper half plane, consisting of $j$ triangles. Each vertex has one of two colors, say blue or red. The boundary of $D_{j}$ has one marked "active" edge with one blue endpoint $b_{j}$ and one red endpoint $r_{j}$, along which the growth of $D_{j}$ takes place. Initially $(j=0)$ there are no triangles, we color the nonpositive integers blue, the positive integers red, and set the active edge as the line segment $[0,1]$. To pass from $D_{j}$ to $D_{j+1}$, we glue a triangle to $D_{j}$ along the marked edge. There are four possible ways of gluing the new triangle, depicted by an explicit example in Figure 5. We label these four choices as $B, b, R$, and $r$. 


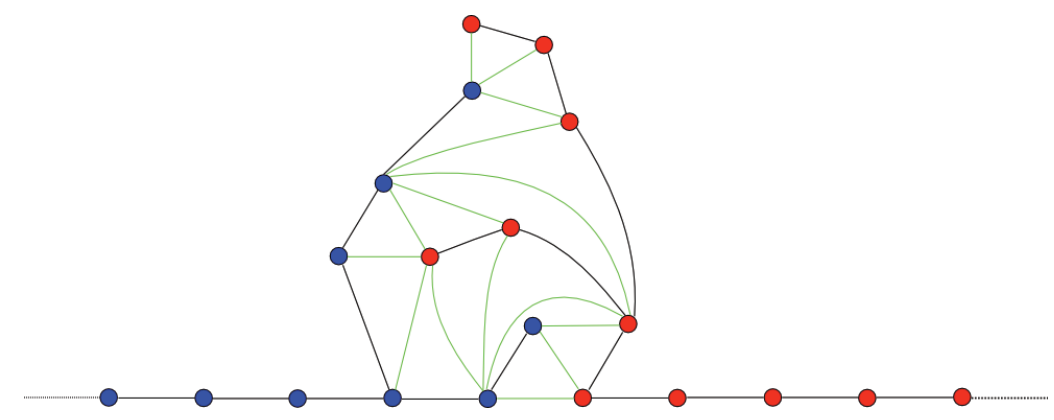

FiguRE 5. The upper half plane necklace for the sequence $B R b R R b B B r r R B R R$.

Choice $B$ consists of adding a new blue vertex $b_{j+1} \in \mathbb{H} \backslash D_{j}$, adding two edges $\left(b_{j+1}, b_{j}\right)$ and $\left(b_{j+1}, r_{j}\right)$, and setting $r_{j+1}=r_{j}$. Thus $\left(b_{j+1}, r_{j}\right)$ is the new active edge, and $D_{j+1}$ is the union of $D_{j}$ with the triangle $t_{j}$ with vertices $b_{j}, r_{j}, b_{j+1}$. Similarly, choice $R$ consists of adding a red vertex $r_{j+1}$, adding the edges $\left(r_{j+1}, b_{j}\right)$ and $\left(r_{j+1}, r_{j}\right)$, and setting $b_{j+1}=b_{j}$. Choice $b$ involves two slightly different cases: in the case that $b_{j} \in \mathbb{H}$, consists of letting $b_{j+1}$ be the counterclockwise neighbor of $b_{j}$ in the boundary of $D_{j}$, setting $r_{j+1}=r_{j}$, and adding the new active edge $b_{j+1}, r_{j+1}$. In the other case that $b_{j}$ is an integer $m$, we set $b_{j+1}=m-1$, add the edge $[m, m-1]$, again set $r_{j+1}=r_{j}$, and again add the new active edge $b_{j+1}, r_{j+1}$. In either case, a triangle with vertices $\left(b_{j+1}, b_{j}, r_{j+1}\right)$ is added. Similarly, choice $r$ sets $r_{j+1}$ as the clockwise neighbor of $r_{j}$ (and sets $r_{j+1}=m+1$ and adds the edge $[m, m+1]$ if $r_{j}=m$ is a positive integer), sets $b_{j+1}=b_{j}$, and adds the edge $\left(b_{j}, r_{j+1}\right)$.

Definition 3.1. Given a finite word $X=x_{1} x_{2} \ldots x_{n}$ in the letters $x_{i} \in\{B, b, R, r\}$, we denote by $T_{+}(X)$ the disc triangulation $D_{n}$ obtained by successively applying the choices $x_{i}$. Similarly, we denote by $T_{-}(X)$ the triangulation we obtain if we add triangles to the lower half plane instead of the upper half plane. Both $T_{+}$ and $T_{-}$are rooted triangulations, whose root is the first triangle constructed. We denote by $T(X, k)$ the rooted triangulation obtained from $T_{+}(X)$ by choosing the triangle created at $x_{k}$ as the root.

Note the following simple fact, which is key to our approach. Write $B^{\prime}=b$, $R^{\prime}=r, b^{\prime}=B$, and $r^{\prime}=R$. If $X=x_{1} x_{2} \ldots x_{n}$ and $Y=y_{1} y_{2} \ldots y_{m}$, and if $Z=Z(X, Y)=y_{m}^{\prime} y_{m-1}^{\prime} \ldots y_{1}^{\prime} x_{1} x_{2} \ldots x_{n}$, then

$$
T(Z, m+1)=T_{+}(X) \cup T_{-}(Y) .
$$

Here we interpret the right-hand side as a triangulation of the set $D_{n}(X) \cup$ $D_{m}(Y)$ with root triangle the root of $D_{n}(X)$. We would like to extend the definition to infinite sequences $X=x_{1} x_{2} \ldots$ in a natural way. Notice however that the corresponding graph is not necessarily even locally finite. We will show below that in the probabilistic setting, almost surely such pathologies do not occur. If $X$ and $Y$ are two random infinite sequences with respect to the uniform measure 
(that is, the $x_{i}$ are i.i.d. with $P\left(x_{i}=x\right)=1 / 4$ for all $x \in\{B, b, R, r\}$ ), then we can think of $T_{+}(X)$ and $T_{-}(Y)$ as random triangulated half-planes ("quantum disks"), and interpret $T_{+}(X) \cup T_{-}(Y)$ as a gluing of two independent quantum disks. In the proof of Corollary 1.3 we will show that $T_{+}(X) \cup T_{-}(Y)$ is indeed a disc triangulation. Provide $T_{+}(X) \cup T_{-}(Y)$ with a Riemann surface structure by gluing equilateral triangles as in Section 2.2, and denote the resulting surface by $R(X, Y)$. Our main result of this section is a proof of a conjecture of Scott Sheffield:

Theorem 3.2. The random Riemann surface $R(X, Y)$ is almost surely parabolic.

In order to apply Theorem 1.1, we would like to construct a sequence of random finite disc triangulations converging to $T_{+}(X) \cup T_{-}(Y)$ in distribution. This will be achieved by

$$
T_{n}=T(X, k)
$$

where $X=x_{1} \ldots x_{n}$ is uniform and $k$ is uniformly chosen from $\{1,2, \ldots, n\}$.

Lemma 3.3. The law of $T_{n}$ converges in distribution to the law of $T_{+}(X) \cup T_{-}(Y)$ with respect to $d_{c}$.

The proof of the lemma is not difficult (see Section 3.2.3), but nontrivial. The discussion of Example 2.6 showed that the analog of Lemma 3.3 is false if the infinite necklace is replaced by the 7-regular graph.

3.2.2. Estimates. By means of (3.1), many questions about $T_{n}$ can be reduced to questions about $T_{+}(X)$. With each $X=x_{1} x_{2} x_{3} \ldots$ we associate a function $k \mapsto S_{k} \in \mathbb{Z}^{2}$ as follows: $S_{0}=(0,0)$, and $S_{k+1}=S_{k}+a$, where $a=(1,0),(-1,0),(0,1),(0,-1)$ according to $x_{k+1}=B, b, R, r$. If $X$ is uniformly random, then $S_{k}$ is a simple random walk.

Write $S_{k}=\left(X_{k}, Y_{k}\right)$. We will now examine properties of $T_{+}(X)$ by studying the corresponding properties of the simple random walk. We start by exploring the distribution of the degrees of the vertices. Suppose a blue vertex $v$ was created at time $k$. At that time, it is connected to two edges, so that $\operatorname{deg}(v)=2$. At a later time $j>k$, an additional edge will connect to $v$ if and only if $X_{i} \geq X_{k}$ for all $k \leq i \leq j$, and either $X_{j}=X_{k}$ or $X_{j-1}=X_{k}$ (or both). In other words, a new edge connects to $v$ for each time $j$ at which the walk $X_{j}$ lands on $X_{k}$ or goes from $X_{k}$ to $X_{k}+1$. At the first time $j$ where the walk goes from $X_{k}$ to $X_{k}-1$, the newly formed edge separates $v$ from the boundary of $D_{j}$ so that $v$ becomes an interior vertex and cannot be connected to any edge that is created in the future. A similar analysis applies to red vertices. In particular, the vertex $v=0$ is in the outer boundary (=the boundary minus $\mathbb{Z})$ of $T_{+}\left(x_{1} x_{2} \ldots x_{k}\right)$ if and only if

$$
X_{j} \geq 0 \quad \text { for all } 1 \leq j \leq k .
$$

Similarly, the degree of $v=0$ in $T_{+}\left(x_{1} x_{2} \ldots x_{k}\right)$ satisfies

$$
\operatorname{deg}(0) \leq 2\left|\left\{0 \leq j \leq k: b_{j}=0\right\}\right|=2 \mid\left\{j \leq k: X_{j}=0, X_{i} \geq 0 \text { for all } i<j\right\} \mid .
$$

We use this to show exponential decay of the law of the degrees of the vertices: 
Lemma 3.4. For each $n$, each $j \in\{1,2, \ldots, n\}$ and each of the vertices $v$ of the $j$-th face of $T_{n}$, we have

$$
\mathbb{P}[\operatorname{deg}(v) \geq m] \leq 2\left(\frac{3}{4}\right)^{m / 4} .
$$

Proof. The degrees of the vertices of $T_{n}=T(X, k)$ are obviously independent of the choice $k$ of the root triangle.

By (3.1), we can write

$$
T_{n}=T\left(x_{1} \ldots x_{n}\right)=T_{+}\left(x_{j} \ldots x_{n}\right) \cup T_{-}\left(x_{j-1}^{\prime} \ldots x_{1}^{\prime}\right),
$$

so that the vertices of the $j$-th face of $T_{n}$ appear as the vertices of the root faces of $T_{+}\left(x_{j} \ldots x_{n}\right)$ and $T_{-}\left(x_{j-1}^{\prime} \ldots x_{1}^{\prime}\right)$. We assume that the vertex $v$ of the face $f_{j}\left(T_{n}\right)$ corresponds to the vertex 0 of $T_{+}\left(x_{j} \ldots x_{n}\right)$ (the other two cases are similar). Then, if $d_{+}$and $d_{-}$denote the degrees of $v$ in $T_{+}\left(x_{j} \ldots x_{n}\right)$ and in $T_{-}\left(x_{j-1}^{\prime} \ldots x_{1}^{\prime}\right)$, we have $\operatorname{deg}(v) \leq d_{+}+d_{-}$. It follows that

$$
\mathbb{P}_{n}[\operatorname{deg}(v) \geq m] \leq 2 \mathbb{P}\left[d_{+} \geq m / 2\right] .
$$

By (3.3), we have

$$
\mathbb{P}\left[d_{+} \geq k / 2\right] \leq \mathbb{P}\left[\mid\left\{j \leq m: X_{j}=0, X_{i} \geq 0 \text { for all } i<j\right\} \mid \geq m / 4\right] \leq\left(\frac{3}{4}\right)^{m / 4} .
$$

Next, we show that the boundary of $T_{n}$ is small compared to $n$.

Lemma 3.5. For all $t>0$,

$$
\mathbb{P}\left[\left|\partial T_{n}\right| \geq t \sqrt{n}\right] \leq 8 \exp \left(-\frac{t^{2}}{72}\right) .
$$

Proof. Since the boundary of $T(X, k)$ does not depend on $k$, the claim is equivalent to the claim

$$
\mathbb{P}\left[\left|\partial T_{+}\left(x_{1} \ldots x_{n}\right)\right| \geq t \sqrt{n}\right] \leq 8 \exp \left(-\frac{t^{2}}{72}\right) .
$$

A blue vertex $v=b_{k}$ is in the boundary if either $v$ is a negative integer $m$, in which case $X_{k}=m$, or if $v$ is not an integer and $X_{j} \geq X_{k}$ for all $k<j \leq n$. Writing $a=\left|\min _{1 \leq k \leq n} X_{k}\right|$ and $b=\left|\min _{1 \leq k \leq n} Y_{k}\right|$, it follows that

$$
\left|\partial T_{+}\left(x_{1} \ldots x_{n}\right)\right| \leq a+b+\left|X_{n}+a\right|+\left|Y_{n}+b\right| .
$$

Thus, by symmetry,

$$
\mathbb{P}\left[\left|\partial T_{+}\left(x_{1} \ldots x_{n}\right)\right| \geq t \sqrt{n}\right] \leq 4 \mathbb{P}\left[a \geq \frac{t}{6} \sqrt{n}\right] \leq 8 \mathbb{P}\left[X_{n} \leq-\frac{t}{6} \sqrt{n}\right] .
$$

By Azuma's inequality,

$$
\mathbb{P}\left[X_{n} \leq-\frac{t}{6} \sqrt{n}\right] \leq \exp \left(-\frac{(t / 6)^{2}}{2}\right),
$$

and the claim follows. 
Finally, we conclude that, with large probability, the root has large distance from the boundary:

Lemma 3.6. Let $r$ be the root vertex. There is a constant $C$ such that

$$
\mathbb{P}\left[d\left(r, \partial T_{n}\right) \leq k\right] \leq \frac{(C \log n)^{k+1}}{\sqrt{n}} .
$$

In particular, for each fixed $k$,

$$
\mathbb{P}\left[d\left(r, \partial T_{n}\right) \leq k\right] \rightarrow 0 \quad \text { as } n \rightarrow \infty .
$$

Proof. Let

$$
M_{n}=\max \left\{\operatorname{deg}(v): v \text { vertex of } T_{n}\right\}
$$

By Lemma 3.4,

$$
\mathbb{P}\left[M_{n} \geq k\right] \leq \sum_{v \in T_{n}} \mathbb{P}[\operatorname{deg}(v) \geq k] \leq n\left(\frac{3}{4}\right)^{k / 4} \leq \frac{1}{n}
$$

if $k \geq C \log n$ with $C=8 / \log (4 / 3)$. By Lemma 3.5 there is $C$ such that

$$
\mathbb{P}\left[\left|\partial T_{n}\right|>C \sqrt{n \log n}\right]<\frac{1}{n} .
$$

On the event $\left\{M_{n} \leq C \log n ;\left|\partial T_{n}\right| \leq C \sqrt{n \log n}\right\}$ we have

$$
\left|\left\{v: d\left(v, \partial T_{n}\right) \leq k\right\}\right| \leq\left|\partial T_{n}\right|(C \log n)^{k} \leq \sqrt{n}(C \log n)^{k+1} .
$$

Since every triangle appears equally likely as the root triangle,

$$
\mathbb{P}\left[d\left(r, \partial T_{n}\right) \leq k\right] \leq \frac{2}{n}+\frac{(C \log n)^{k+1}}{\sqrt{n}}
$$

and the lemma follows.

\subsubsection{Parabolicity of the infinite necklace.}

Proof of Corollary 1.3. We wish to apply Theorem 1.1 to $T_{+}(X) \cup T_{-}(Y)$ and thereby prove parabolicity of the Riemann surface $R(X, Y)$. To this end, we will realize $T_{+}(X) \cup T_{-}(Y)$ as the distributional limit of the random rooted finite unbiased disc triangulations $T_{n}$. Lemma 3.6 immediately implies that $d\left(o_{n}, \partial T_{n}\right) \rightarrow \infty$ in law. It remains to show Lemma 3.3 and one-endedness.

To show Lemma 3.3, write $\left.T_{+}(X) \cup T_{-}(Y)\right|_{m}$ for the disc triangulation given by only using the first $m$ elements of $X$ and $Y$, namely

$$
\left.T_{+}(X) \cup T_{-}(Y)\right|_{m}=T_{+}\left(x_{1}, x_{2}, \ldots, x_{m}\right) \cup T_{-}\left(y_{1}, y_{2}, \ldots, y_{m}\right) .
$$

By the discussion before Lemma 3.4, almost surely the ball of radius $r$ around the root is determined at a finite time,

$$
B_{r}\left(\left.T_{+}(X) \cup T_{-}(Y)\right|_{m}\right)=B_{r}\left(\left.T_{+}(X) \cup T_{-}(Y)\right|_{m^{\prime}}\right)
$$


for all $m, m^{\prime}$ large. This gives a rigorous definition of the random triangulation $T_{+}(X) \cup T_{-}(Y)$ and thus the Riemann surface $R(X, Y)$. Fix a possible rooted combinatorial ball $B$ of radius $r$. We need to show that

$$
\mathbb{P}\left[B_{r}\left(T_{n}\right)=B\right] \rightarrow \mathbb{P}\left[B_{r}\left(T_{+}(X) \cup T_{-}(Y)\right)=B\right]
$$

as $n \rightarrow \infty$. It follows from (3.8) that

$$
\left|\mathbb{P}\left[B_{r}\left(T_{+}(X) \cup T_{-}(Y)\right)=B\right]-\mathbb{P}\left[B_{r}\left(\left.T_{+}(X) \cup T_{-}(Y)\right|_{m}\right)=B\right]\right|<\epsilon
$$

for every $\epsilon$ and $m \geq m_{\epsilon}$. With $T_{n}=T\left(x_{1} \cdots x_{n}, k\right)$, since $\mathbb{P}[m<k<n-m]=$ $1-2 m / n$ we therefore have, by (3.1),

$$
\left|\mathbb{P}\left[B_{r}\left(T_{+}(X) \cup T_{-}(Y)\right)=B\right]-\mathbb{P}\left[B_{r}\left(T_{n}\right)=B\right]\right|<\epsilon+\frac{2 m}{n},
$$

and the lemma follows.

To show that $T_{+}(X) \cup T_{-}(Y)$ is one ended, fix a finite subgraph $H$ and choose $m$ so large that $H$ is contained in the interior of the finite disc triangulation $D=$ $\left.T_{+}(X) \cup T_{-}(Y)\right|_{m}$. The complement of $D$ is connected (through $\partial D$ ), hence $T_{+}(X) \cup$ $T_{-}(Y) \backslash H$ has only one infinite connected component.

Now Theorem 1.1 shows that $R(X, Y)$ is almost surely parabolic.

\section{References}

[1] Angel, O. and Schramm, O.: Uniform infinite planar triangulations. Comm. Math. Phys. 241 (2003), 191-213.

[2] Astala, K., Jones, P., Kupiainen, A. and Saksman, E.: Random conformal weldings. Acta Math. 207 (2011), 203-254.

[3] Benjamini, I.: Random planar metrics. In Proceedings of the International Congress of Mathematicians, vol. IV, 2177-2187. Hindustan Book Agency, New Delhi, 2010.

[4] Benjamini, I. And Curien, N.: On limits of graphs sphere packed in Euclidean space and applications. European J. Combin. 32 (2011), 975-984.

[5] Benjamini, I. and Schramm, O.: Recurrence of distributional limits of finite planar graphs. Electron. J. Probab. 6 (2001), 1-13.

[6] Bouttier, J., Di Francesco, P. And Guitter, E.: Planar maps as labeled mobiles. Electron. J. Combin. 11 (2004), Research Paper 69, 27 pp.

[7] Duplantier, B. And Sheffield, S.: Liouville quantum gravity and KPZ. Invent. Math. 185 (2011), no. 2, 333-393.

[8] Guriel-Gurevich, O. and Nachmias, A.: Recurrence of planar graph limits. Ann. of Math. (2) 177 (2013), no. 2, 761-781.

[9] He, Z. H. And Schramm, O.: Hyperbolic and parabolic packings. Discrete Comput. Geom. 14 (1995), 123-149.

[10] Le Gall, J.-F.: The topological structure of scaling limits of large planar maps. Invent. Math. 169 (2007), 621-670.

[11] Le Gall, J.-F. And Paulin, F.: Scaling limits of bipartite planar maps are homeomorphic to the 2-sphere. Geom. Funct. Anal 18 (2008), 893-918. 
[12] McCaughan, G.: A recurrence/transience result for circle packings. Proc. Amer. Math. Soc. 126 (1998), 3647-3656.

[13] Miermont, G.: On the sphericity of scaling limits of random planar quadrangulations. Electron. Commun. Probab. 13 (2008), 248-257.

[14] Rohde, S.: Oded Schramm: from circle packings to SLE. Ann. Probab. 39 (2011), no. 5, 1621-1667.

[15] Schatffer, G.: Conjugaison d'arbres et cartes combinatoires alatoires. Ph. D. Thesis, University of Bordeaux, 1998.

[16] Schramm, O.: Transboundary extremal length. J. Anal. Math. 66 (1995), 307-329.

[17] Sheffield, S.: Conformal weldings of random surfaces: SLE and the quantum gravity zipper. Preprint available at arXiv:1012.4797 [math.PR] .

[18] Sheffield, S.: Quantum gravity and inventory accumulation. Preprint available at arXiv:1108.2241 [math.PR].

[19] Stephenson, K.: Introduction to circle packing. The theory of discrete analytic functions. Cambridge University Press, Cambridge, 2005.

Received November 30, 2011.

James T. Gill: Saint Louis University, Department of Mathematics and Computer Science, 220 N. Grand Blvd., St. Louis, MO 63108, USA.

E-mail: jgill5@slu.edu

Steffen Rohde: University of Washington, Department of Mathematics, Box 354350, Seattle, WA 98195, USA.

E-mail: rohde@math.washington.edu

The first author was supported by NSF Grant DMS-1004721, and the second author by NSF Grant DMS-0800968. 